\title{
Stellar coronagraphy: laboratory testing of the Achromatic Interfero Coronagraph
}

\author{
J. P. Rivet ${ }^{1}$, J. Gay ${ }^{1}$, J. Dalmau ${ }^{1}$, G. Cassar $^{1}$ and Y. Rabbia ${ }^{2}$ \\ ${ }^{1}$ Observatoire de la Côte d'Azur, B.P. 4229, F-06304 Nice Cedex 4, France \\ email: rivet@obs-nice.fr \\ ${ }^{2}$ Observatoire de la Côte d'Azur, Avenue Copernic, F-06130 Grasse, France
}

\begin{abstract}
We present new laboratory measurements of the intrinsic rejection performances in infrared $(1.9 \mu \mathrm{m}$ to $2.5 \mu \mathrm{m})$ for a prototype of Achromatic Interfero Coronagraph (AIC). We first recall basics about the AIC, then describe the prototype under consideration. We give detailed explanations about the experimental setup and the procedures followed to measure the rejection rate. We end up with a discussion of the results obtained.
\end{abstract}

Keywords. instrumentation: miscellaneous.

\section{Basics on the standard AIC}

The Achromatic Interfero-Coronagraph (AIC) has been introduced by Gay \& Rabbia (1996) (see also Baudoz et al., 2000) as a new concept of stellar coronagraphs. Instead of rejecting the light of a central star by a more or less smoothly varying complex transmission in the focal plane of a telescope, the AIC rejects the light by destructive interferences. The device works like a modified Michelson's interferometer (see Figure 1): the input light is divided by a beam splitter cube into two balanced beams leaving at right angle. The first one passes through the "passive" arm (thick solid lines on Figure 1), which involves only flat mirrors. The second one enters the "phase shifting" arm (thick dashed lines on Figure 1). The latter arm involves a cat's eye structure with a primary parabolic mirror and a small secondary spherical mirror, so that the light beam passes through a focus. Crossing a focal point is known to lead to an achromatic $\pi$ phase shift for the amplitude (see Gouy, 1890 and Born \& Wolf, 1999), and to a central symmetry of the image. So, when both beams recombine, the light of a star lying on the axis (thus invariant by central symmetry) cancels because of the $\pi$ phase shift, provided both arms of the interferometer have equal optical length. Cancellation does not occur for off-axis sources (see Figure 2).

The entrance pupil of the telescope has to be symmetric, and the odd-order aberrations (turbulence, optical parts) have to be as low as possible (Baudoz et al. (2000)). Extra input and output optics (sketched as lenses in Figure 1) are required to fit the AIC between a telescope and its IR detector.

\section{The prototype under consideration}

The prototype used for this experiment is a compact monolithic device based upon a bloc of fused silica with a cavity drilled inside for the phase shifting arm. The beam splitter cube, the parabolic mirror and the spacer containing the cavity for the passive arm are also made out of silica, and glued onto the main bloc (see Figure 3 ). 


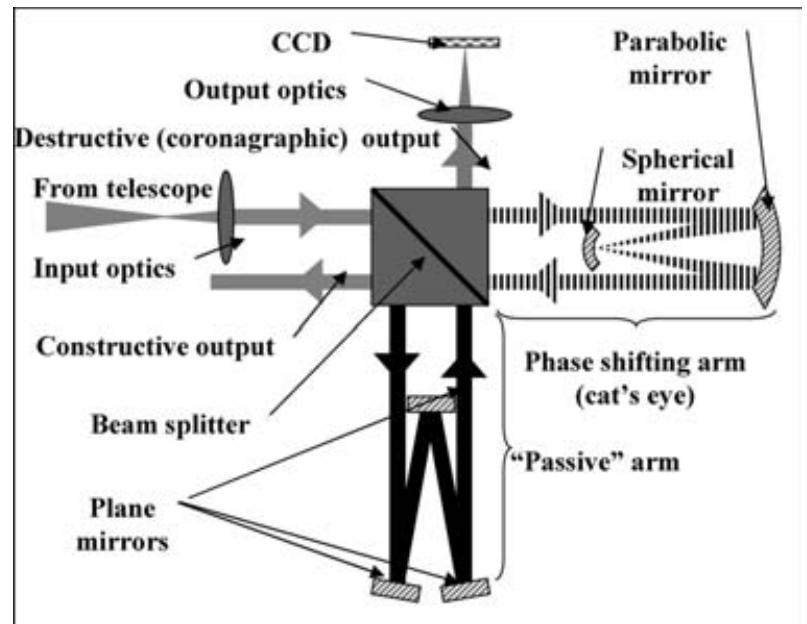

Figure 1. The configuration of the standard AIC.

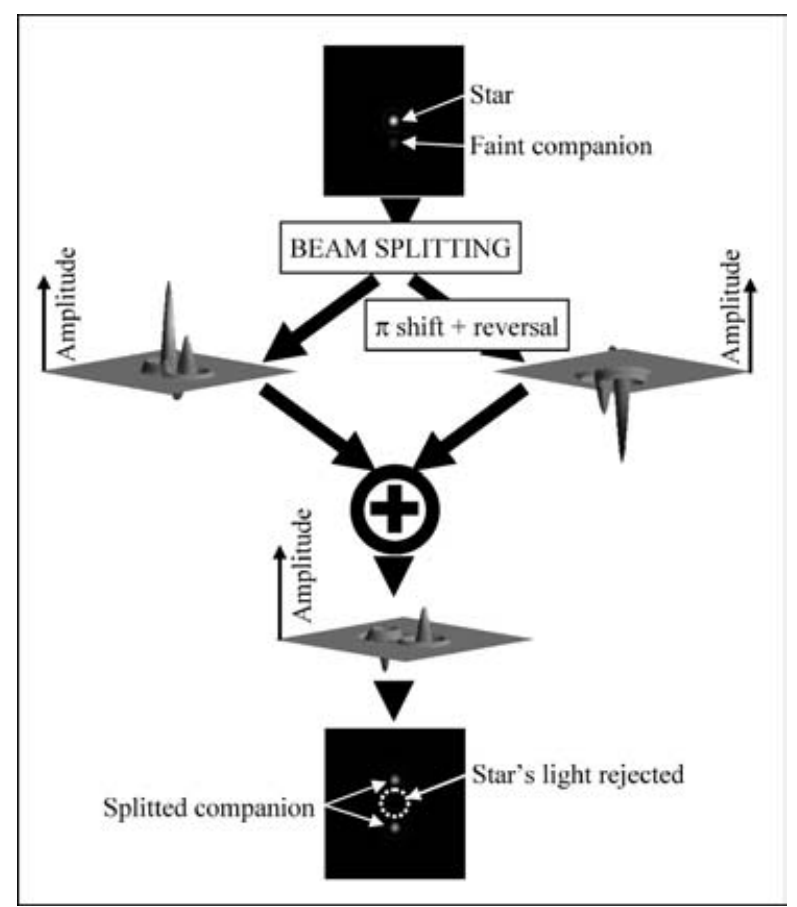

Figure 2. The principle of the AIC.

Since the equality of the geometrical lengths of both arms cannot be guaranteed to the required accuracy, the cavities are filled with dry nitrogen, and a suitable pressure difference is imposed, so as to compensate for the geometrical length difference $(\simeq 5 \mu \mathrm{m})$.

\section{The experiment}

This prototype, with a suitable input and output optics, has been inserted within the focal environment of the $3.6 \mathrm{~m}$ telescope at $\mathrm{CFH}$ (Hawaii), and tested on the sky. The 


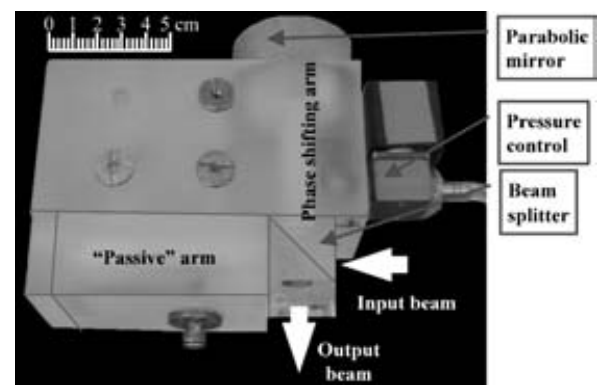

Figure 3. The prototype of AIC.

rejection performances were limited by the residual atmospheric turbulence, and possibly also by the quality of the optical components of the AIC (mainly the parabolic mirror).

To evaluate the intrinsic limitations of the AIC itself, a laboratory experiment has been conducted to measure the rejection rate of this prototype. The optical bench layout is sketched in Figure 4. The pinhole source diameter is $50 \mu \mathrm{m}$; the pupil stop diameter is $5.4 \mathrm{~mm}$, and the focal length of the BK7 collimator is $106 \mathrm{~cm}$ in the IR band under consideration $(1.9 \mu \mathrm{m}$ to $2.5 \mu \mathrm{m})$. The $Z n S e$ objective lens focuses the output beam on the receptor, a CEDIP Jade IR camera, with a Germanium filter.

Several parameters have to be very carefully fine tuned to achieve optimal rejection rates: The $X Y$ position of the pupil stop, the $Z$ position of the collimator lens and of the objective lens, the nitrogen pressure difference, and the $X Y$ position of the source.

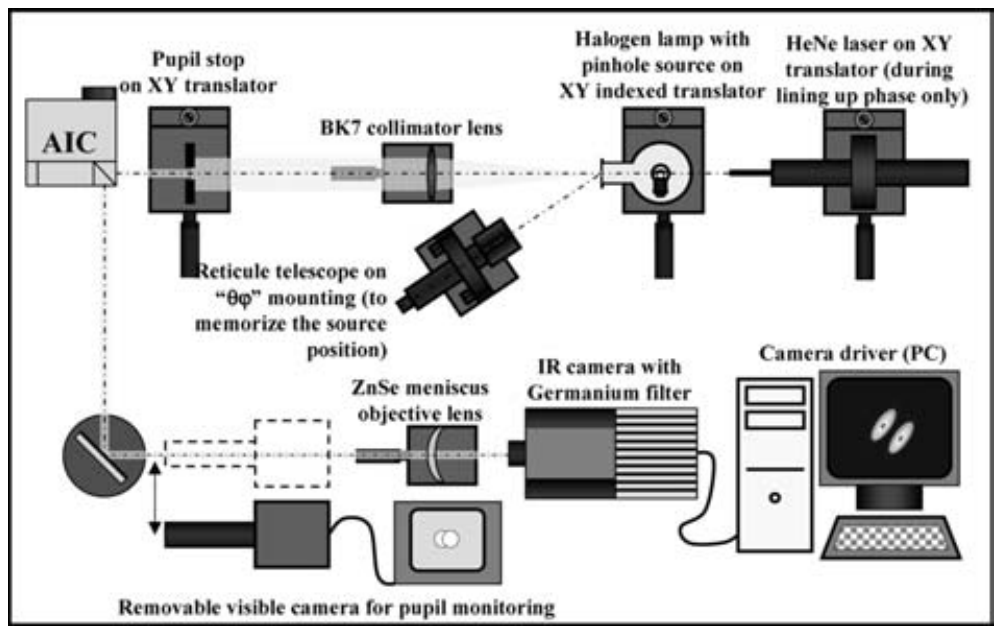

Figure 4. The experimental setup for rejection measurements.

\section{The fine tuning procedures}

The $X Y$ position of the pupil stop is tuned "manually", so as to make the two images of the pupil coincide on the visible camera monitor. For the other parameters, the "manual" tuning has revealed unsatisfactory, and the following more accurate procedure has been used :

- one first obtains a coarse grained value of the parameter.

- Then, one explores systematically several values around it. 
- For each value, one obtains the IR intensity field by averaging over 64 suitably pre-processed snapshots, and one measures the peak intensity value at the center of the field.

- One fits the curve "value of the parameter versus peak intensity" with a parabola, and extract the optimal value of the parameter, corresponding to the minimum of the peak intensity.

After a complete fine tuning cycle for all the relevant parameters, the residual IR intensity can be measured by averaging over 512 snapshots.

\section{The measured rejection rate}

The coronagraphic rejection rate is the ratio of the input energy for an on-axis source, to the residual output energy. The input energy is evaluated by pushing the source far out of the axis. The most carefully processed data led to the following result:

$$
\text { Measured rejection rate } \simeq 500 \text { for } \lambda \in[1.9 \mu m-2.5 \mu m]
$$

\section{The extinction profile}

The normalized residual energy, when studied as a function of the source offset, yields the extinction profile. Figure 5 shows the theoretical extinction profile (solid curve), and the measured data (dots).

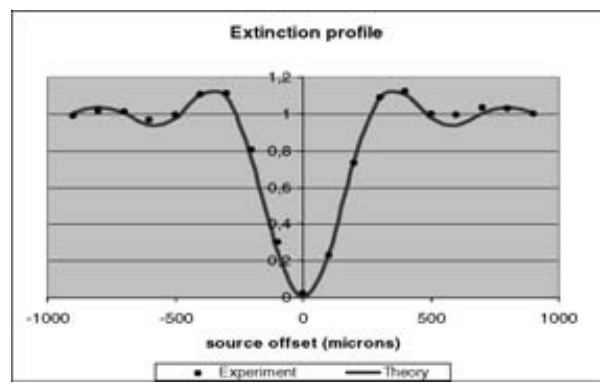

Figure 5. The rejection profile of the AIC (rejection versus distance from axis). Solid line: theoretical prediction; Dots: measured values.

\section{Conclusion}

Despite the poor quality of its parabolic mirror, the AIC prototype at hand has led to a measured rejection rate close to $\mathbf{5 0 0}$ over a relatively large bandwidth, even with a non-totally coherent source. Further on-sky tests at CFHT are now required to fully qualify this prototype for scientific applications.

\section{References}

Gay, J. \& Rabbia, Y. 1996, C.R. Acad. Sci. Paris II 322, 265

Baudoz, P., Rabbia, Y., \& Gay, J. 2000, Astr. Astroph. Suppl. Series 141, 319

Gouy, L.G. 1890, C.R. Acad. Sci. Paris 110, 1251

Born, M. \& Wolf, E. 1999, (Cambridge University Press), p. 499 